\title{
Associated risk factors and arterial occlusions in patients with von Willebrand disease: Analysis of the literature and report of two cases
}

\author{
Girolami A, Ferrari S, Bertomoro A, Peroni E, Sambado L, Casonato A \\ Department of Medicine, University of Padua Medical School, Padua, Italy. \\ Correspondence: Antonio Girolami. Address: Department of Medicine, University of Padua Medical School, Via Ospedale \\ Civile 105, Padua, Italy. Email: antonio.girolami@unipd.it
}

Received: March 15, 2014

DOI : $10.5430 /$ crim.v1n2p135

Online Published: May 16, 2014

URL: http://dx.doi.org/10.5430/crim.v1n2p135

\begin{abstract}
Objective: To investigate the features of all reported cases of arterial occlusion which occurred in patients with Von Willebrand Disease.

Patients and methods: Personal records were re-evaluated together with a time-unlimited Pubmed search using pertinent keywords. Occlusive events had to be documented by objective means. All cases of myocardial infarction and other acute coronary syndromes were considered. Cases of angina were instead excluded.

Results: 56 patients with arterial occlusion were gathered from the literature (44 cases of acute coronary syndromes and 12 cases of ischemic stroke. Two additional cases were obtained from personal records ( 1 case of myocardial infarction and 1 case of unstable angina) for a total of 58 patients. Peripheral arterial thrombosis has never been described in von Willebrand disease patients. The majority of these patients presented common risk factors such as smoking, hypertension, diabeties, dyslipidemia, obesity. Replacement therapy, contrary to what seen in hemophilias, had instead a minor role if any.

Conclusions: von Willebrand disease is unable to protect from atherosclerosis and atherothrombosis. The common risk factors for arterial disease such as hypertension, diabetiesdyslipidemia and smoking are able to neutralize the potential protective effect of VW Factor deficiency. Despite this, the prevalence of arterial occlusions in von Willebrand disease appears decreased in comparison with the general populations at least in some countries.
\end{abstract}

\section{I ntroduction}

Von Willebrand disease (VWD) is, together with the hemophilias, the most frequent congenital bleeding disorder. Usually it is an autosomal dominant disorder originally described in Scandinavian countries but subsequently demonstrated to be diffuse worldwide. An autosomal recessive form has been also described. The condition is classified in several subgroups, each with some peculiar laboratory features (see Table1) ${ }^{[1,2]}$.

Bleeding tendency is variable but, often, especially in Type 1 and Type 3, severe. 
Table 1. Main features of von Willebrand Disease types.

\begin{tabular}{|c|c|c|c|c|c|c|}
\hline Type & Defect & $\begin{array}{l}\text { VWF: } \\
\text { RCo }\end{array}$ & $\begin{array}{l}\text { Ristocetin-induced } \\
\text { platelet aggregation } \\
\text { (RIPA) }\end{array}$ & $\begin{array}{l}\text { Multimeric } \\
\text { Pattern }\end{array}$ & $\begin{array}{l}\text { Bleeding } \\
\text { tendency }\end{array}$ & $\begin{array}{l}\text { Platelet } \\
\text { VWF }\end{array}$ \\
\hline $\begin{array}{l}\text { Type } \\
1\end{array}$ & $\begin{array}{l}\text { Partial quantitative } \\
\text { deficiency of VWF }\end{array}$ & Decreased & Decreased & $\begin{array}{l}\text { Uniform } \\
\text { decrease: } \\
\text { all present }\end{array}$ & Variable & $\begin{array}{l}\text { Normal/ } \\
\text { Reduced }\end{array}$ \\
\hline $\begin{array}{l}\text { Type } \\
2 \mathrm{~A}\end{array}$ & $\begin{array}{l}\text { Defective platelet-dependent } \\
\text { VWF functions }\end{array}$ & Decreased & Decreased/Absent & $\begin{array}{l}\text { Decrease of } \\
\text { large multimers }\end{array}$ & $\begin{array}{l}\text { Moderate/ } \\
\text { Severe }\end{array}$ & $\begin{array}{l}\text { Normal/ } \\
\text { Reduced }\end{array}$ \\
\hline $\begin{array}{l}\text { Type } \\
\text { 2B }\end{array}$ & $\begin{array}{l}\text { Increased platelet-dependent } \\
\text { VWF interaction }\end{array}$ & Decreased & Increased & $\begin{array}{l}\text { Decrease of } \\
\text { large multimers }\end{array}$ & $\begin{array}{l}\text { Moderate/ } \\
\text { Severe }\end{array}$ & Normal \\
\hline $\begin{array}{l}\text { Type } \\
2 \mathrm{M}\end{array}$ & $\begin{array}{l}\text { Defective platelet-dependent } \\
\text { VWF functions }\end{array}$ & $\begin{array}{l}\text { Decreased/ } \\
\text { Absent }\end{array}$ & Decreased/Absent & $\begin{array}{l}\text { All multimers } \\
\text { present }\end{array}$ & Moderate & $\begin{array}{l}\text { Normal/ } \\
\text { Reduced }\end{array}$ \\
\hline $\begin{array}{l}\text { Type } \\
2 \mathrm{~N}\end{array}$ & $\begin{array}{l}\text { Defective VWF binding to } \\
\text { FVIII }\end{array}$ & Normal & Normal & Normal & $\begin{array}{l}\text { Mild/ } \\
\text { Moderate }\end{array}$ & Normal \\
\hline $\begin{array}{l}\text { Type } \\
3\end{array}$ & $\begin{array}{l}\text { Severe deficiency of VWF } \\
\text { and moderate FVIII } \\
\text { deficiency }\end{array}$ & Absent & Absent & Undetectable & Severe & Absent \\
\hline
\end{tabular}

The main bleeding manifestations are mucosal and cutaneous, epistaxis, gastro-intestinal bleeding, hematomas. In severe forms hemarthrosis has also been reported.

It is commonly accepted that patients with congenital bleeding disorders in general, including VWD, are protected from thrombosis and atherosclerosis. However, during the last few years this assumption has been challenged since several patients with a congenital bleeding disorder and a thrombotic event have been reported. As far as VWD is concerned a few cases with either arterial or venous thrombosis have been described ${ }^{[3-6]}$. Furthermore, it has been shown that the defect does not protect from the occurrence of atherosclerosis ${ }^{[4,7]}$.

Recently at least two papers reported a reduction of arterial thrombosis among the patients with VWD as compared with the general population ${ }^{[8,9]}$. The purpose of the present study is to analyze all cases of VWD patients with arterial thrombosis described in the literature together with the presentation of two new personal cases.

\section{Patients and methods}

The clinical charts, the out-patient clinic records, the follow-up notes and the laboratory results of all 486 patients with VWD studied in Padua during the years 1972-2010 were reevaluated.

All forms of VWD were included and classified according to the decision of the authors of the single papers (see Table 1).

Myocardial infarction (MI) had to be proven by enzyme elevation, compatible ECG changes and coronary angiography.

Other acute coronary syndromes such as non ST elevation myocardial infarction (NSTEMI) and unstable angina were also included providing patients had undergone coronary angiography and coronary invasive procedures, namely percutaneous transluminal coronary angioplasty (PTCA), stent implantation, coronary artery by-pass graft (CABG). 
Cases with stable angina or effort angina were excluded even if documented by angiography. Ischemic stroke had to be demonstrated by CAT or MRI or by angiography. Transitory ischemic attacks (TIAs) were excluded.

Peripheral arterial lesions had to be documented by angiography.

The presence of commons risk factor for arterial occlusions, namely; diabeties, hypertension, smoking, obesity, dyslipidemia were recorded together with all other potentially thrombophilic causes. Finally the replacement therapy being given to the patients at the time of the thrombotic event was also always recorded whenever reported.

\section{Results}

The evaluation of the records dealing with the VWD patients studied in Padua yielded two new cases, one case of M.I. and one case of unstable angina.

\subsection{Case 1}

The patient is a 75 year old female, with a VWD type 2B diagnosed at the age of 38 when she presented an excessive bleeding tendency during and after a cholecystectomy. The FVIII level was 78\% (normal range 60\%-160\%), VWF Antigen (VWF: Ag) 62.6\% (normal range 60\%-160\%) and Von Willebrand factor ristocetin cofactor (VWF: RCo), 22.1\% (normal range 60\%-130\%). The absence of high molecular weight multimers (HMWM) was also documented. Previously she had shown occasional epistaxis and was treated with Desmopressin or VWF reach FVIII concentrates for prolonged epistaxis and bleeding after tooth extractions. Since the age of 52 she has been treated for hypertension with verapamil and a thiazide diuretic but cholesterol was always normal. At the age of 63 she started complaining of claudicatio due to diffuse atherosclerosis of lower limbs arteries extending to the aorta. Carotid artery atherosclerosis was also present but less severe.

A brain computerised axial tomography (CAT) showed ischemic lesions. The atherosclerotic lesions of the legs worsened despite accepted therapy and gangrene of the fifth right toe developed. She underwent hyperbaric treatment with some improvement Because of the worsering of the ischemic lesions the patient underwent a complete re-evaluation which led to the discovery of a retroperitoneal leiomyosarcoma. The patient underwent radiation therapy (26 sessions), with shrinking of the tumour mass. A few months after the completion of the radiotherapy, the patient presented with a severe chest pain and died in the Coronary Unit, despite all therapeutic attempts, because of a massive anterolateral and inferior M.I.The patients had never previously lamented chest pain or presented ECG alterations.

\subsection{Case 2}

A 69-year-old male with type Vicenza VWD (R1205H mutation) presented with a chronic picture compatible with unstable angina. He was a smoker and known to have angina due to diffuse coronary artery disease (CAD) since the age of 55. At the age of 60 he had already undergone a CABG. He had also a type 2 diabetes and high cholesterol. Basal blood tests showed factor VIII level of 34\% (normal range 60\%-160\%), VWF Antigen (VWF: Ag) of 17.8\% (normal range 60-160) and Von Willebrand factor ristocetin cofactor (vWF: RCo) of $16.6 \%$ (normal range $60 \%-130 \%$ ). He had a past history of several muco-cutaneous bleeding episodes. Other relevant past history events were: chemotherapy for a nasal non Hodgkin lymphoma, a surgically treated testicular lymphoma, and a HCV-related hepatocellular carcinoma treated with transarterialchemoembolizzation (TACE).

After admission, coronary angiography via radial artery puncture demonstrated severe and extensive CAD. During the procedure unfractionated heparin (1000 IU) was administered intra catheter. Prophylaxis with F VIII/VWF concentrates (40 IU/Kg) was administered before and for 3 days after the endovascular procedure. Due to the extension of the atherosclerotic lesions, endovascular or surgical treatment for CAD was not deemed feasible. 
Considering the high cardiovascular risk and worsening status, clopidogrel was administered for secondary anti-platelet prophylaxis. Since the patient had never taken antiplatelet drug before, and considering his lifelong bleeding history, a long- term prophylaxis with VWF concentrates was planned. To avoid excessive F VIII concentrations and the correlated potential risk of arterial and venous thrombosis, a highly-purified plasma derived VWF concentrate (Wilfactin, LFB) was preferably used. The patient received $60 \mathrm{UI} / \mathrm{kg}$, twice weekly to maintain VWF: Ag and VWF: RCo above 25\%-30\% and FVIII below $80 \%-100 \%$. Anti hemorrhagic prophylaxis, in association with antiplatelet treatment, was given for 7 months. No bleeding or thrombotic complications were observed during a further 7-month follow-up. The evaluation of the literature has allowed the gathering of 56 additional cases of arterial thrombosis (see Table 2) ${ }^{[9-22]}$. The total number is therefore 58.The breakdown figures were the following: M.I (including NSTEMI), 39 cases; unstable angina, 7 patients, ischemic stroke, 12 cases. There was no patient with a peripheral artery occlusion. Age varied between 32 and 81 (mean 58.2). 14 were female whereas, 16 were male. In 28 cases, gender was not reported.

Associated risk factors were present in 28 out of the 58 patients. However in 20 instances the presence of risk factors was not reported or dealt with. Therefore the actual prevalence of risk factors was 27 out of 29 patients (see Figure 1). Informations about the therapeutic management were supplied in 35 cases. A conservative approach without invasive corrective procedures but which included the use of antiplatelet drugs, heparins or coumadin was followed in 8 patients.

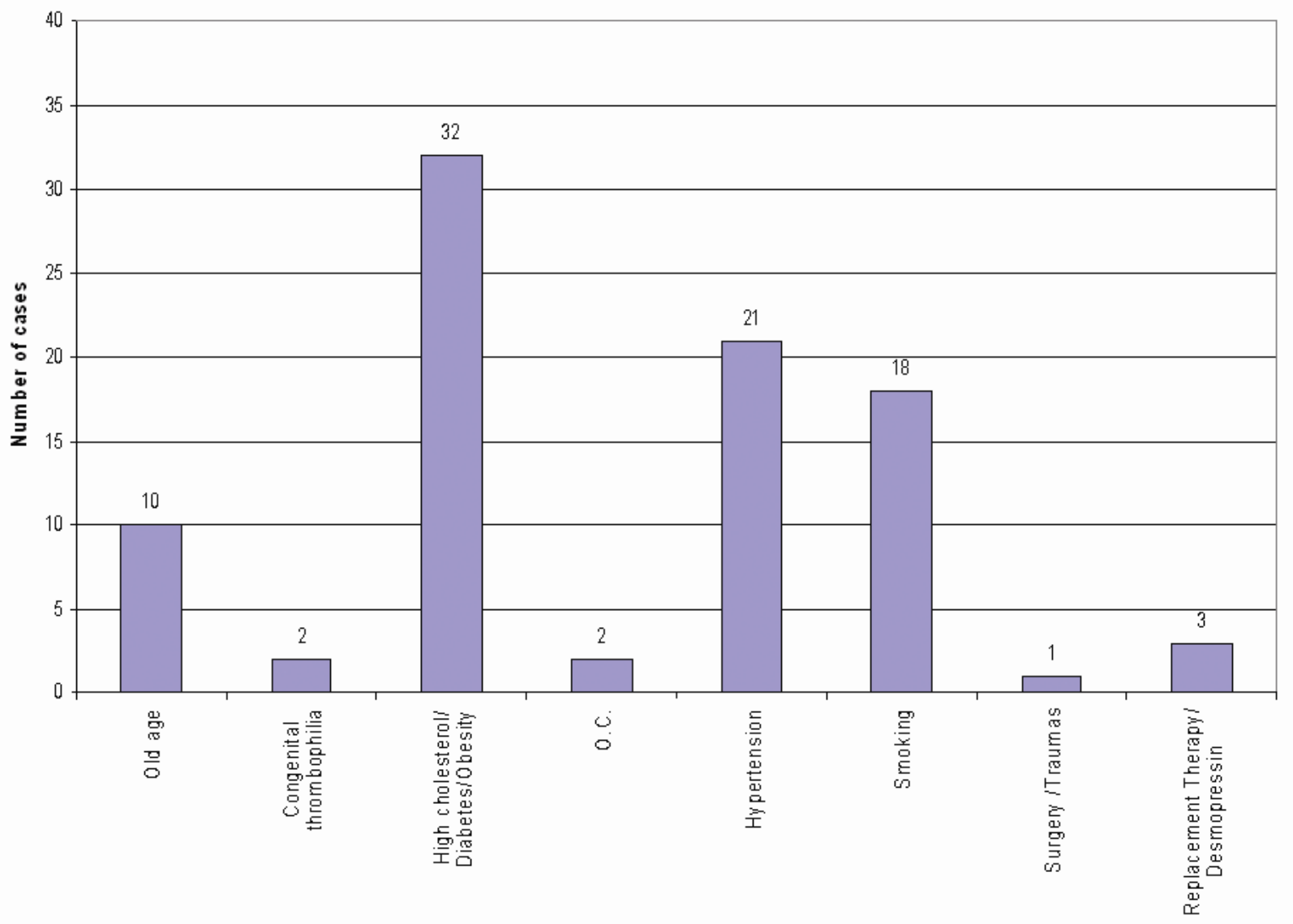

Figure 1. Risk factors present in 28 patients with VWD and arterial occlusions: Several patients had more than one risk factor. No risk factors were present or no informations were supplied for the remaining patients. The low number of replacement therapy associated thrombotic events is probably biased since in some studies patients who developed thrombosis after substitution therapy were excluded or no mention was made about its potential causative role. O.C: Oral contraceptives. 
Invasive corrective or surgical procedures were carried out in 27 patients. No detail could be gathered for the remaining patients. The evalution of the condition was reported only in 21 cases. It was fair or good in 17 cases. It was good in one patient and there were 3 fatalities.

\section{Discussion}

The occurrence of a thrombotic or atherosclerotic arterial occlusion in patients with a congenital bleeding tendency represents always a striking event. It was commonly and often naively maintained that congenital hypocoagulability could protect from both atherosclerosis and thrombosis.

Autopsy studies had demonstrated a few decades ago that severe atherosclerosis changed could occur in patients with VWD $^{[3,4]}$. More recently, occasional reports have shown that both arterial and venous thrombosis events may occur in these patients ${ }^{[6,23]}$.

These observations do not necessarily indicate that the defect does not protect from thrombosis. It indicateds only that the protection is not absolute. The same has been demonstrated for the hemophilias and other congenital clotting factor deficiencies, notably F VII deficiency ${ }^{[24-28]}$.

Comparison of the prevalence of thrombotic event seen in hemophilias with that observed in VWD is not easily obtainable. Hemophilia A or B are commonly accepted to be present in 1 out of 5000 males or in 1 out of 10.000 subjects of both genders whereas the prevalence of VWD is not surely established even though it is commonly accepted, for symptomatic patients, to be 1 out of 10.000 people ${ }^{[29,30]}$. Furthermore, VWD, contrary to the hemophilia, is a complex condition with many variant subtypes ${ }^{[1,31,32]}$.The overall impression is that the VWD is associated with a decreased incidence of arterial thrombosis.

A recent study has shown a clear reduction of arterial occlusions in Dutch patients with VWD as compared with two control groups representing the general population ${ }^{[9]}$.

All these Dutch patients, belonging to different types of VWD presented also common risk factors for arterial disease. A high incidence of common risk factors for arterial thrombosis are present also in other patients with VWD and thrombosis. Therefore it seems that the clotting defect is unable to neutralize the effect of these common risk factors.

In another study, on 98 patients mainly type 1 and mainly female, 8 cases of CAD, probably MI, and 5 cases of ischemic stroke ${ }^{[8]}$ were observed. Unfortunately the study did not deal with the individual features of the single patients but compared the rough incidence of events with that seen in a suited control population. The study concluded also for a descreased incidence of arterial thrombosis in VWD in comparison with the general population ${ }^{[8]}$.

Apparently there is no type of VWD particularly prone to have thrombotic complications. The reported cases reflect the entire gamut of subtypes even though the majority (about 70\%) belong to type 1 . This roughly reflects the normal distribution of VWD subtypes. On theoretical grounds it could be surmised that the types which lack HMWM could represent an added risk since these patients may have undergone platelets activation by the HMW multimers. The amount of intra-platelet VWF could also play a role. The two cases studied in Padua had normal amounts of intra platelets VWF. Unfortunately the measurement of platelet VWF is seldom carried out and therefore no conclusion can be drawn. The most striking observation of this paper is the high prevalence of the common risk factors for atherosclerosis present in almost all patients with VWD and arterial thrombosis.

Several patients had more the one risk factor and smoking, diabetes and hypertension were those most widely represented (see Table 2). 
Table 2. Myocardial infarction and other arterial occlusions in patients with VWD as reported in the literature.

\begin{tabular}{|c|c|c|c|c|c|c|c|}
\hline $\begin{array}{l}\text { Authors, } \\
\text { Year }\end{array}$ & $\begin{array}{l}\text { Age, } \\
\text { gender }\end{array}$ & $\begin{array}{l}\text { Type of } \\
\text { defect }\end{array}$ & $\begin{array}{l}\text { Type of } \\
\text { thrombosis }\end{array}$ & $\begin{array}{l}\text { Bleeding } \\
\text { tendency }\end{array}$ & $\begin{array}{l}\text { Associated risk } \\
\text { factors }\end{array}$ & $\begin{array}{l}\text { Therapy and } \\
\text { evolution }\end{array}$ & Comments \\
\hline \multicolumn{8}{|l|}{$\begin{array}{l}\text { Silwer et al., } \\
\text { (1966) }\end{array}$} \\
\hline Case 1 & $69, \mathrm{~F}$ & I & MI & severe & n.r. & n.r.; fatal & $\begin{array}{l}\text { significant } \\
\text { atherosclerosis at } \\
\text { autopsy }\end{array}$ \\
\hline Case 2 & $72, \mathrm{M}$ & I & MI & moderate & hypertension & n.r.; fatal & $\begin{array}{l}\text { Some as above; } \\
\text { the patients had } \\
\text { also a stroke }\end{array}$ \\
\hline \multicolumn{8}{|l|}{$\begin{array}{l}\text { Moss } \\
\text { etal., (1986) }\end{array}$} \\
\hline & $63, \mathrm{~F}$ & n.r. & UA & moderate & $\begin{array}{l}\text { Diabetes } \\
\text { hypertension }\end{array}$ & $\begin{array}{l}\text { CABG; } \\
\text { good }\end{array}$ & \\
\hline \multicolumn{8}{|l|}{$\begin{array}{l}\text { Goodnough } \\
\text { et al., (1983) }\end{array}$} \\
\hline Case 1 & $53, \mathrm{~F}$ & n.r. & stroke & moderate & $\begin{array}{l}\text { Post } \\
\text { hysterectomy }\end{array}$ & & \\
\hline Case 2 & $81, \mathrm{M}$ & n.r. & MI & severe & n.r. & n.r., n.r. & \\
\hline Case 3 & $62, \mathrm{M}$ & n.r. & MI & n.r. & n.r. & CABG; fair & \\
\hline Case 4 & $54, \mathrm{M}$ & n.r. & MI & n.r. & n.r. & n.r., n.r. & \\
\hline Case 5 & $52, \mathrm{M}$ & n.r. & MI & n.r. & n.r. & n.r., n.r. & \\
\hline \multicolumn{8}{|l|}{$\begin{array}{l}\text { Dulhoste } \\
\text { et al., (1983) }\end{array}$} \\
\hline Case 1 & $36, \mathrm{M}$ & I & MI & moderate & $\begin{array}{l}\text { smoking, high } \\
\text { cholesterol; fair }\end{array}$ & n.r., n.r. & $\begin{array}{l}\text { Angiographically } \\
\text { proven }\end{array}$ \\
\hline Case 2 & $51, \mathrm{M}$ & I & MI & Severe & $\begin{array}{l}\text { smooking, high } \\
\text { cholesterol; fair }\end{array}$ & n.r., n.r. & \\
\hline $\begin{array}{l}\text { Slaughter } \\
\text { et al., (1993) }\end{array}$ & $51, \mathrm{M}$ & undefined & MI & n.r. & n.r. & $\begin{array}{l}\text { CSBG,ASA; } \\
\text { good }\end{array}$ & $\begin{array}{l}\text { suspected type } 2 \\
\text { variant }\end{array}$ \\
\hline $\begin{array}{l}\text { Wong } \\
\text { et al., (1996) }\end{array}$ & $69, \mathrm{~F}$ & n.r. & MI & Mild & n.r. & $\begin{array}{l}\text { rTPA, PTCA, } \\
\text { ASA, heparin; } \\
\text { fair }\end{array}$ & $\begin{array}{l}\text { Restenosis two } \\
\text { months later led to } \\
\text { stent implantation }\end{array}$ \\
\hline $\begin{array}{l}\text { Fragasso } \\
\text { et al., (1998) }\end{array}$ & $61, \mathrm{M}$ & I & MI & Mild & n.r. & $\begin{array}{l}\text { rtPA, heparin; } \\
\text { good }\end{array}$ & \\
\hline $\begin{array}{l}\text { Arjomand } \\
\text { et al., (2002) }\end{array}$ & $45, \mathrm{~F}$ & I & MI & Severe & $\begin{array}{l}\text { smoking, } \\
\text { hypertension }\end{array}$ & $\begin{array}{l}\text { PTCA, stents; } \\
\text { good }\end{array}$ & \\
\hline $\begin{array}{l}\text { James } \\
\text { et al., (2002) }\end{array}$ & $70, \mathrm{~F}$ & I & MI & moderate & $\begin{array}{l}\text { smoking, } \\
\text { hypertension }\end{array}$ & $\begin{array}{l}\text { PTCA; } \\
\text { good }\end{array}$ & $\begin{array}{l}\text { Patient had also } \\
\text { scleroderma }\end{array}$ \\
\hline $\begin{array}{l}\text { Basso } \\
\text { et al,.(2004) }\end{array}$ & $50, \mathrm{M}$ & $2 \mathrm{~A}$ & MI & moderate & $\begin{array}{l}\text { aFVII, smoking, } \\
\text { Diabetes type 1, } \\
\text { hypertension }\end{array}$ & $\begin{array}{l}\text { Conservative; } \\
\text { fair }\end{array}$ & $\begin{array}{l}\text { Patient had also } \\
\text { angiodysplasia }\end{array}$ \\
\hline $\begin{array}{l}\text { Franchini } \\
\text { et al., (2004) }\end{array}$ & $53, \mathrm{M}$ & I & MI & n.r. & $\begin{array}{l}\text { smoking, } \\
\text { hypertension, } \\
\text { high colesterol, } \\
\text { Het prothrombin } \\
\text { G20210A }\end{array}$ & $\begin{array}{l}\text { PTCA, stents; } \\
\text { n.r. }\end{array}$ & \\
\hline
\end{tabular}


Table 2. (continued)

\begin{tabular}{|c|c|c|c|c|c|c|c|}
\hline $\begin{array}{l}\text { Authors, } \\
\text { Year }\end{array}$ & $\begin{array}{l}\text { Age, } \\
\text { gender }\end{array}$ & $\begin{array}{l}\text { Type of } \\
\text { defect }\end{array}$ & $\begin{array}{l}\text { Type of } \\
\text { thrombosis }\end{array}$ & $\begin{array}{l}\text { Bleeding } \\
\text { tendency }\end{array}$ & $\begin{array}{l}\text { Associated risk } \\
\text { factors }\end{array}$ & $\begin{array}{l}\text { Therapy and } \\
\text { evolution }\end{array}$ & Comments \\
\hline $\begin{array}{l}\text { Grainge } \\
\text { et al., (2006) }\end{array}$ & $32, \mathrm{~F}$ & I & stroke & mild & $\begin{array}{l}\text { Desmopressism } \\
\text { oking, oral } \\
\text { contraception }\end{array}$ & ASA; fair & $\begin{array}{l}\text { Previous four } \\
\text { pregnancies } \\
\text { without } \\
\text { complications }\end{array}$ \\
\hline $\begin{array}{l}\text { Mc Donald } \\
\text { et al., (2006) }\end{array}$ & $56, \mathrm{M}$ & I & MI & mild & n.r. & $\begin{array}{l}\text { PTCA, drug } \\
\text { eluting stent; } \\
\text { good }\end{array}$ & NSTEMI \\
\hline $\begin{array}{l}\text { De Wee } \\
\text { et al., (2008) }\end{array}$ & $52, \mathrm{~F}$ & I or $2 \mathrm{M}$ & stroke & mild & $\begin{array}{l}\text { Desmopressin } \\
\text { for arthroscopy }\end{array}$ & n.r., n.r. & $\begin{array}{l}\text { Patient had also } \\
\text { bilateral } \\
\text { pulmonary } \\
\text { embolism }\end{array}$ \\
\hline \multicolumn{8}{|l|}{$\begin{array}{l}\text { Lim and } \\
\text { Pruthi (2012) }\end{array}$} \\
\hline Case 1 & $74, \mathrm{~F}$ & I & ACS & n.r. & n.r. & $\begin{array}{l}\text { U.H. } \\
\text { ASA; good }\end{array}$ & $\begin{array}{l}\text { Single institution } \\
\text { cases. Patients } \\
\text { who had received } \\
\text { replacement } \\
\text { therapy during the } \\
\text { week prior to the } \\
\text { actual event were } \\
\text { excluded }\end{array}$ \\
\hline Case 2 & 59, M & $2 \mathrm{M}$ & ACS & $\begin{array}{l}\text { Probably } \\
\text { severe }\end{array}$ & n.r. & $\begin{array}{l}\text { No (due to } \\
\text { bleeding); fair }\end{array}$ & \\
\hline Case 3 & $80, F$ & $2 B$ & ACS & n.r & n.r & CABG; good & \\
\hline Case 4 & $65, \mathrm{M}$ & I & ACS & n.r & n.r & $\begin{array}{l}\text { PCI, stent, ASA, } \\
\text { clopidogrel; } \\
\text { good }\end{array}$ & \\
\hline Case 5 & $65, \mathrm{~F}$ & I & ACS & n.r & n.r & CABG; fair & \\
\hline Case 6 & $74, \mathrm{M}$ & I & ACS & n.r & n.r & $\begin{array}{l}\text { CABG,ASA; } \\
\text { good }\end{array}$ & \\
\hline Case 7 & $55, \mathrm{~F}$ & I & ACS & n.r & n.r & ASA; fair & \\
\hline \multirow[t]{2}{*}{$\begin{array}{l}\text { Qureshi } \\
\text { et al.,(2012) }\end{array}$} & n.r., n.r, & I & $\begin{array}{l}8 \text { cases of } \\
\text { M.I. }\end{array}$ & n.r & n.r & n.r., n.r. & $\begin{array}{l}\text { No individual data } \\
\text { on patients are } \\
\text { supplied; patients } \\
\text { mainly female }\end{array}$ \\
\hline & n.r., n.r, & I & $\begin{array}{l}6 \text { cases of } \\
\text { stroke }\end{array}$ & n.r & n.r & n.r., n.r. & \\
\hline $\begin{array}{l}\text { Rathore } \\
\text { et al., (2013) }\end{array}$ & $46, F$ & I & MI & moderate & $\begin{array}{l}\text { diabetes, } \\
\text { hypertension, } \\
\text { high } \\
\text { cholesterole, } \\
\text { smoking }\end{array}$ & $\begin{array}{l}\text { PTCA, stent , } \\
\text { ASA,clopidogre } \\
\text { l; good }\end{array}$ & $\begin{array}{l}\text { NSTEMI; } \\
\text { previous balloon } \\
\text { angioplasty }\end{array}$ \\
\hline \multicolumn{8}{|l|}{$\begin{array}{l}\text { Sanders } \\
\text { et al., (2013) }\end{array}$} \\
\hline Case 1 & 52 n.r. & I & MI & n.r. & $\begin{array}{l}\text { smoking, } \\
\text { hypertension, } \\
\text { high cholesterol, } \\
\text { diabetes }\end{array}$ & $\begin{array}{l}\text { CABG, ASA; } \\
\text { n.r. }\end{array}$ & $\begin{array}{l}\text { Multicentric study } \\
\text { in the } \\
\text { Netherlands. Data } \\
\text { gathered from } \\
\text { questionnaires. }\end{array}$ \\
\hline
\end{tabular}


Table 2. (continued)

\begin{tabular}{|c|c|c|c|c|c|c|c|}
\hline $\begin{array}{l}\text { Authors, } \\
\text { Year }\end{array}$ & $\begin{array}{l}\text { Age, } \\
\text { gender }\end{array}$ & $\begin{array}{l}\text { Type of } \\
\text { defect }\end{array}$ & $\begin{array}{l}\text { Type of } \\
\text { thrombosis }\end{array}$ & $\begin{array}{l}\text { Bleeding } \\
\text { tendency }\end{array}$ & $\begin{array}{l}\text { Associated risk } \\
\text { factors }\end{array}$ & $\begin{array}{l}\text { Therapy and } \\
\text { evolution }\end{array}$ & Comments \\
\hline Case 2 & 48 n.r. & $2 \mathrm{~B}$ & MI & n.r. & $\begin{array}{l}\text { smoking, } \\
\text { obesity, } \\
\text { hypertension }\end{array}$ & PTCA; n.r. & \\
\hline Case 3 & 75 n.r. & $2 \mathrm{~A}$ & MI & n.r. & $\begin{array}{l}\text { smoking, } \\
\text { hypertension, } \\
\text { high cholesterol }\end{array}$ & $\begin{array}{l}\text { CABG, ASA; } \\
\text { n.r. }\end{array}$ & \\
\hline Case 4 & 60 n.r. & I & MI & n.r. & $\begin{array}{l}\text { smoking, } \\
\text { hypertension, } \\
\text { high cholesterol }\end{array}$ & $\begin{array}{l}\text { Two CABG, } \\
\text { ASA; n.r. }\end{array}$ & $\begin{array}{l}\text { Previously had } \\
\text { V.A. }\end{array}$ \\
\hline Case 5 & 64 n.r. & $2 \mathrm{~A}$ & MI & n.r. & $\begin{array}{l}\text { smoking, } \\
\text { obesity, } \\
\text { hypertension, } \\
\text { high cholesterol }\end{array}$ & $\begin{array}{l}\text { Two CABG, } \\
\text { PTCA; } \\
\text { dypiridamol; } \\
\text { n.r. }\end{array}$ & $\begin{array}{l}\text { Previously had } \\
\text { V.A. }\end{array}$ \\
\hline Case 6 & 66 n.r. & I & MI, UA & n.r. & $\begin{array}{l}\text { smoking, } \\
\text { hypertension, } \\
\text { diabetes, high } \\
\text { cholesterol }\end{array}$ & $\begin{array}{l}\text { PTCA, } \\
\text { U.H.,ASA; n.r. }\end{array}$ & \\
\hline Case 7 & 77 n.r & I & UA & n.r & $\begin{array}{l}\text { hypertension, } \\
\text { high cholesterol }\end{array}$ & PTCA; n.r. & \\
\hline Case 8 & 59n.r. & $2 \mathrm{~B}$ & UA & n.r. & $\begin{array}{l}\text { smoking, } \\
\text { hypertension, } \\
\text { high cholesterol }\end{array}$ & $\begin{array}{l}\text { PTCA, two } \\
\text { CABG,clopidog } \\
\text { rel; n.r. }\end{array}$ & \\
\hline Case 9 & 50n.r. & $2 \mathrm{~A}$ & UA & n.r. & $\begin{array}{l}\text { smoking, high } \\
\text { cholesterol }\end{array}$ & $\begin{array}{l}\text { CABG, ASA; } \\
\text { n.r. }\end{array}$ & \\
\hline Case10 & 67, n.r. & $2 \mathrm{~A}$ & UA & n.r. & $\begin{array}{l}\text { smoking,hypert } \\
\text { ension, high } \\
\text { cholesterol }\end{array}$ & $\begin{array}{l}\text { Two PTCA, } \\
\text { ASA, } \\
\text { clopidogrel; n.r. }\end{array}$ & $\begin{array}{l}\text { Previous PTCA at } \\
57\end{array}$ \\
\hline Case 11 & 46, n.r. & $2 \mathrm{~A}$ & UA & n.r. & $\begin{array}{l}\text { hypertension, } \\
\text { Prot. S } \\
\text { deficiency }\end{array}$ & $\begin{array}{l}\text { PTCA, ASA; } \\
\text { n.r. }\end{array}$ & \\
\hline Case12 & 59, n.r. & I & UA & n.r. & $\begin{array}{l}\text { smoking, high } \\
\text { cholesterol }\end{array}$ & $\begin{array}{l}\text { CABG, ASA } \\
\text {;n.r. }\end{array}$ & \\
\hline Case13 & 62, n.r. & I & UA & n.r. & $\begin{array}{l}\text { smoking,obesity } \\
\text { hypertension, } \\
\text { high cholesterol, } \\
\text { diabetes }\end{array}$ & $\begin{array}{l}\text { CABG, } \\
\text { coronary } \\
\text { endarterectomy; } \\
\text { n.r. }\end{array}$ & $\begin{array}{l}\text { Patient had also a } \\
\text { TIA }\end{array}$ \\
\hline Case 14 & 59, n.r & I & stroke & n.r. & $\begin{array}{l}\text { smoking, } \\
\text { obesity, } \\
\text { hypertension }\end{array}$ & $\begin{array}{l}\text { Coronary } \\
\text { endarterectomy } \\
\text { Coumadin; n.r. }\end{array}$ & $\begin{array}{l}\text { Case n. } 16 \text { in } \\
\text { original paper }\end{array}$ \\
\hline \multicolumn{8}{|c|}{$\begin{array}{l}\text { Present paper } \\
(2013)\end{array}$} \\
\hline & $75, \mathrm{~F}$ & $2 \mathrm{~B}$ & MI & severe & hypertension; & $\begin{array}{l}\text { Only } \\
\text { supportive; } \\
\text { fatal; }\end{array}$ & Died in C.U. \\
\hline & 69, M & I & UA & Moderate & $\begin{array}{l}\text { Hypertension, } \\
\text { Diabetes type I, } \\
\text { smoking }\end{array}$ & $\begin{array}{l}\text { ASA, } \\
\text { clopidogrel; } \\
\text { poor }\end{array}$ & $\begin{array}{l}\text { Coronary } \\
\text { angiography } \\
\text { excluded } \\
\text { procedures of } \\
\text { stenting or by } \\
\text { passing }\end{array}$ \\
\hline
\end{tabular}


The effect of replacement therapy seems less important as compared with the hemophilias ${ }^{[24,26]}$. However, this cannot be stated with certainty since in some studies ${ }^{[8,17]}$, patients who had developed the thrombotic event after replacement therapy were excluded or no mention was made about it potential causative role. In only one study it was specifically stated that no thrombotic event occurred after replacement therapy ${ }^{[9]}$.

Furthermore, it has to be noted that VWD patients receive less frequently prophylactic treatment as compared with hemophiliac.

An observation on the prevalence of the arterial occlusion is also indicated. The prevalence of arterial occlusion found in our series ( 2 out of 486 patients, namely $0.41 \%$ ) is quite different from that observed among Dutch or American patients (20 cases out of 635 patients or 14 cases out of 198 patients, namely 3.15\% or 7.07\% respectively).

This discrepancy may have several explanations but probably it is due mainly to the lower fat diet usually followed in Italy as compared to that followed in the Netherlands and the United States. The observation indicates, for the first time, the existence of potential regional differences in the incidence of arterial occlusions, not only in "normal" subjects, but also in patients with congenital bleeding disorders.

The fact that coronary arteries are more frequently involved than other arterial districts confirms the observation already made for hemophilia patients ${ }^{[24,26]}$. No hemophilia patients has been described with a peripheral artery occlusion. The same seems true for VWD. These observations indicate the existence of discrepancies in the susceptibility to atherothrombosis of different arterial districts ${ }^{[33]}$. Due to the limited number of patients studied so far, no definitive remarks can be made on this subject. In this regard it is important to note that an anti VW factor approach has been recently proposed in the prophylaxis of ischemic stroke ${ }^{[34]}$. A finding that will surely be the object of future research.

In conclusion, this study, once again, underscores the pivotal role played by the known risk factors in the pathogenesis of atherosclerosis and atherothrombosis. It is clear that even severe congenital coagulation defects are unable to prevent the occurrence of atherosclerotic lesion and their consequent occlusive complications.

This has practical clinical implications. Physicians caring for patients with a congenital bleeding disorder should always keep in mind that these patients, usually predisposed to bleeding, may also, occasionally, present a thrombotic event.

\section{Acknowledgements}

All papers regardless of the language, dealing with patients with VWD who presented an arterial occlusion were obtained through a time unlimited Pubmed search. Several pertinent keywords were used together with the Medical Subject Headings (MESH) proposed by Pubmed. The searchs were carried out in January 2006 and again in September 2013. Details Tabs reported in the Pubmed search were also checked in every instance. Original Papers were then obtained with the help of the Pinali Medical library of our University. Cross-checking of the references listed at the end of the single papers was also carried out to avoid omissions.

\section{References}

[1] Sadler JE, Budde U, Eikenboom JC, Favaloro EJ, Hill FG, Holmberg L, et al. Working Party on von Willebrand Disease Classification.Update on the pathophysiology and classification of von Willebrand disease: a report of the Subcommittee on von WillebrandFactor. JThrombHaemost. 2006; 4: 2103-14. http://dx.doi.org/10.1111/j.1538-7836.2006.02146.x

[2] James PD, LillicrapD. The molecular characterization of von Willebrand disease: good in parts.Br J Haematol. 2013; 161: 166-176. http://dx.doi.org/10.1111/bjh.12249

[3] Goodnough LT, Saito H, Ratnoff OD. Thrombosis or myocardial infarction in congenital clotting factor abnormalities and chronic thrombocytopenias: a report of 21 patients and a review of 50 previously reported cases. Medicine. 1983; 62: 248-55. http://dx.doi.org/10.1097/00005792-198307000-00004 
[4] Silwer J, Cronberg S, Nilsson IM. Occurrence of arteriosclerosis in von Willebrand's disease. Acta Med Scand. 1966; 180: 475-84. http://dx.doi.org/10.1111/j.0954-6820.1966.tb02860.x

[5] Franchini M, Veneri D. Are only haemophiliacs protected against ischemic heart disease? ThrombHaemost. 2004; 92: 1455-1455.

[6] Girolami A, Tezza F, Scapin M, Vettore S, Casonato A. Arterial and venous thrombosis in patients with von Willebrand's disease: a critical review of the literature. J Thromb Thrombolysis. 2006; 21: 175-8. http://dx.doi.org/10.1007/s11239-006-5732-0

[7] Federici AB, Mannucci PM, Fogato E, Ghidoni P, Matturri L. Autopsy findings in three patients with von Willebrand disease type IIB and type III: presence of atherosclerotic lesions without occlusive arterial thrombi. ThrombHaemost. 1993; 70: 758-61.

[8] Qureshi W., Hassan S., Dalak V., Kuriakose Ph. Thrombosis in Von WIllebrand disease. Thromb. Res. 2012; 130 : e255-e258. http://dx.doi.org/10.1016/j.thromres.2012.09.005

[9] Sanders Y, Eikenboom J, de Wee E, van der Bom J, Cnossen M, Degenaar-Dujardin M, et al. Reduced prevalence of arterial thrombosis in von Willebrand disease. J Thromb. Haemost. 2013; 11: 845-54. http://dx.doi.org/10.1111/jth.12194

[10] Arjomand H, Aquilina P, McCormick D. Acute myocardial infarction in a patient with von Willebrand disease: pathogenetic dilemmas and therapeutic challenges. J Invasive Cardiol. 2002; 14: 615-8.

[11] Basso IN, Keeling D. Myocardial infarction following recombinant activated factor VII in a patient with type 2A von Willebrand disease. Blood Coagul Fibrinolysis. 2004; 15: 503-4. http://dx.doi.org/10.1097/00001721-200408000-00010

[12] De Wee EM, Ikram MK, Dippel DW, Leebeek FW. Transient focal cerebral ischaemia and bilateral pulmonary embolism after desmopressin treatment for von Willebrand's disease. Haemophilia. 2008; 14: 1133-4. http://dx.doi.org/10.1111/j.1365-2516.2008.01841.x

[13] Dulhoste MN, Bonnet J, Vergnes C, Choussat A, Bricaud H. Maladie de Willebrand et athérosclérosecoronarienne. A propos de troiscas. Arch Mal Coeur Vaiss. 1989; 82: 1875-8.

[14] Fragasso G, Camba L, Pizzetti G, Pagnotta P, Chierchia SL. Successful thrombolysis for acute myocardial infarction in Type I von Willebrand's disease (vWD). Am J Hematol. 1998; 57: 180. http://dx.doi.org/10.1002/(SICI)1096-8652(199802)57:2<180::AID-AJH16>3.0.CO;2-G

[15] Grainge C, NokesT.Cerebral arterial thrombosis in a young woman following vasopressin for von Willebrand's disease. ThrombHaemost. 2005; 93: 380.

[16] James PR, de Belder AJ, Kenny MW. Successful percutaneous transluminal coronary angioplasty for acute myocardial infarction in von Willebrand's disease. Haemophilia. 2002; 8: 826-7. http://dx.doi.org/10.1046/j.1365-2516.2002.00681.x

[17] Lim MY, Pruthi RK. Outcomes of management of acute coronary syndrome in patients with congenital bleeding disorders: a single center experience and review of the literature. Thromb Res. 2012; 130: 316-22. http://dx.doi.org/10.1016/j.thromres.2012.02.050

[18] Macdonald J, Srinivasan M, More R. Percutaneous coronary intervention in a patient with von Willebrand's disease presenting with an acute coronary syndrome. J Invasive Cardiol. 2006; 18: 174-7.

[19] Moss RA, Mena RR, Morin RJ. Atherosclerosis and a coronary artery bypass operation in a woman with von Willebrand disease. West J Med. 1980; 133: 515-7.

[20] Rathore S, Deleon D, Akram H, Sane D, Ball T. Percutaneous coronary intervention and the management of acute coronary syndromes in patients with von Willebrand disease. J Invasive Cardiol. 2013; 25: E81-6.

[21] Slaughter TF, Mody EA, Oldham HN Jr, Reves JG, O’Connor CM, Parker JK, Greenberg CS. Management of a patient with type IIC von Willebrand's disease during coronary artery bypass graft surgery. Anesthesiology. 1993; 78: 195-7. http://dx.doi.org/10.1097/00000542-199301000-00027

[22] Wong CB, Schreiber TL. Acute myocardial infarction in a patient with von Willebrand disease. CathetCardiovascDiagn. 1996; 38: 287-8. http://dx.doi.org/10.1002/(SICI)1097-0304(199607)38:3<287::AID-CCD16>3.0.CO;2-E

[23] Biere-Rafi S, Zwiers M, Peters M, van der Meer J, Rosendaal FR, Büller HR, Kamphuisen PW. The effect of haemophilia and von Willebrand disease on arterial thrombosis: a systematic review. Neth J Med. 2010; 68: 207-14.

[24] Girolami A, Randi ML, Ruzzon E, Zanon E, Girolami B. Myocardial infarction, other arterial thrombosis and invasive coronary procedures, in hemaophilia B: a critical evaluation of reported cases. J Thromb Thrombolysis. 2005; 20: 43-46. http://dx.doi.org/10.1007/s11239-005-2227-3

[25] Girolami A, BertozziI,Berti de Marinis G, Tasinato V, Sambado L. Discrepant ratios of arterial versus venous thrombosis in hemophilia A as compared with hemophilia B. J Thromb Thrombolysis; 2014; 37: 293-297. http://dx.doi.org/ 10.1007/s.11239-013-0943-7

[26] Girolami A, Ruzzon E, Fabris F, Varvarikis C, Sartori R, Girolami B. Myocardial infarction and other arterial occlusions in hemophilia A patients. A cardiological evaluation of all 42 cases reported in the literature. ActaHaematol. 2006; 116: 120-125. http://dx.doi.org/10.1159/000093642 
[27] Girolami A, Tezza F, Scandellari R, Vettore S., Girolami B. Associated prothrombotic condition are probably responsible for the occurence of thrombosis in almost all patients with congenital FVII deficiency. J. Thromb. Thrombol. 2010; 30: 172-178. http://dx.doi.org/10.1007/s11239-009-0435-y

[28] Ruiz-Sáez A. Thrombosis in rare bleeding disorders Hematology. 2012; 17 Suppl 1: 156-158.

[29] Castaman G, Federici AB, Rodeghiero F, MannucciPM.VonWillebrand's disease in the year 2003: towards the complete identification of gene defects for correct diagnosis and treatment. Haematologica. 2003; 88: 94-108.

[30] Franchini M, Coppola A. Atherothrombosis in von Willebrand disease: an analysis of the literature and implications for clinical management. Semin. Thromb. Hemost. 2012; 38: 185-99. http://dx.doi.org/10.1055/s-0032-1301416

[31] Casonato A, Gallinaro L, Cattini MG, Pontara E, Padrini R, Bertomoro A, Daidone V, PagnanA.Reduced survival of type 2B von Willebrand factor, irrespective of large multimer representation or thrombocytopenia. Haematologica. 2010; 95: $1366-72$. http://dx.doi.org/10.3324/haematol.2009.019927

[32] Casonato A, Gallinaro L, Cattini MG, Sartorello F, Pontara E, Padrini R, Bertomoro A, Daidone V, PagnanA.Type 1 von Willebrand disease due to reduced von Willebrand factor synthesis and/or survival: observations from a case series. Transl. Res. 2010; 155: 200-208. http://dx.doi.org/10.1016/j.trsl.2009.12.003

[33] Bilora F, Boccioletti V, Zanon E, Petrobelli F, GirolamiA. Hemophilia A, von Willebrand disease, and atherosclerosis of abdominal aorta and leg arteries: factor VIII and von Willebrand factor defects appear to protect abdominal aorta and leg arteries from atherosclerosis. ClinApplThrombHemost. 2001; 7: 311-3. http://dx.doi.org/10.1177/107602960100700411

[34] De Meyer S, Stole G, Wegner D, Kleinschutz C. Von Willebrand Factor. An emerging target in stroke therapy. Stroke 2012; 43: 599-606. http://dx.doi.org/10.1161/STROKEAHA.111.628867 